\title{
INCREASE IN NOCICEPTIVE INPUT TO SPINOCERVICAL TRACT NEURONS FOLLOWING CHRONIC PARTIAL DEAFFERENTATION ${ }^{1}$
}

\author{
MATHIUS J. SEDIVEC, JANICE OVELMEN-LEVITT, ${ }^{2}$ RUSSELL KARP, AND LORNE M. MENDELL ${ }^{3}$ \\ Department of Neurobiology and Behavior, State Universily of New York at Stony Brook, Stony Brook, New York 11794
}

Received June 21, 1982; Revised December 6, 1982; Accepted February 4, 1983

\begin{abstract}
Recordings from antidromically identified spinocervical tract (SCT) cells in the partially deafferented spinal cord of chloralose-anesthetized cats have revealed reorganization of their input from peripheral receptors in the skin. Immediately after dorsal rhizotomy of segments L5, L6, S1, and S2, (sparing L7), units in L6 had a lower than normal probability of responding to moderate pressure, mechanical nociceptive, noxious heat, and cooling inputs. This persisted for about 6 weeks, after which normal or even larger than normal proportions of SCT units could be driven by these inputs. The proportion of units driven by hair deflection remained large throughout this period. Responses in the L7 segment with intact L7 dorsal root were unchanged. We suggest that afferent fibers from different receptor types differ in the extent of their normal functional projection, accounting for the selective changes after acute deafferentation. The chronic changes may reflect a differential ability of surviving afferent fibers to undergo compensatory changes in their projection (e.g., sprouting) after chronic rhizotomy.
\end{abstract}

Present evidence suggests that deafferentation of the adult cat spinal cord by dorsal rhizotomy results in anatomical and somatotopic reorganization in the dorsal horn. Liu and Chambers (1958) and, more recently, Goldberger and Murray (1974) showed that cutting a number of dorsal roots causes an increase in terminal fields of surviving fibers entering neighboring roots. Studies by Basbaum and Wall (1976) and Pubols and Goldberger (1980) have shown a change in the somatotopic organization of neurons in the partially deafferented dorsal horn. Basbaum and Wall (1976) showed that beginning approximately 30 days after rhizotomy, cells in the deafferented segments exhibit receptive fields outside their dermatome. Pubols and Goldberger (1980) examined dor-

\footnotetext{
${ }^{1}$ This work was supported principally by National Institutes of Health (NIH) Program Project Grant NS14899 (E. R. Perl, principal investigator) and by Research Grant NS16996 to L. M. M. J. O-L. was the recipient of NIH Postdoctoral Training Grant NS06155. We wish to thank Carol Metz at the University of North Carolina at Chapel Hill and Carol Hall at State University of New York (SUNY) at Stony Brook for their excellent technical assistance. We also wish to thank Dr. Alan Light for his helpful comments on the manuscript and Dr. Nancy Mendell for her help with the statistical analysis.

This work was begun at Duke University Medical Center by M. J. S., J. O-L., and L. M. M. and was completed at SUNY at Stony Brook by M. J. S., R. K., and L. M. M.

${ }^{2}$ Present address: Division of Neurosurgery, Duke University Medical Center, Durham, NC 27710.

${ }^{3}$ To whom correspondence should be addressed.
}

sal horn neurons in the lateral portion of the L6 segment that had been partially deafferented by chronic dorsal rhizotomy of all roots caudal to and including L1, sparing L6. They found that cells in the lateral part of the L6 dorsal horn, which normally had large receptive fields with proximal and distal limb components, were partially silenced initially and later recovered responsiveness to distal input which entered the cord through the spared L6 dorsal root. They postulated that partial denervation of cells receiving both proximal and distal input rendered them relatively unresponsive in the acute postoperative period, and that over time, the surviving input from the distal portion of the limb became more potent, possibly as a result of collateral sprouting.

In order to analyze these issues further, we have examined the reorganization of inputs to an identified population of dorsal horn cells, the spinocervical system, using modality rather than somatotopy as the index. Spinocervical tract (SCT) cells exhibit variability in their input (Mendell, 1966; Brown and Franz, 1969), with some being sensitive only to hair deflection or gentle mechanical stimulation. Others appear to receive input from a larger spectrum of peripheral receptor types. This population of cells was chosen because of the ease of recording and identification, which permitted the unambiguous selection of the same class of cells for study in both the normal and the partially deafferented preparations. A preliminary account of this work has been published (Sedivec et al., 1981). 


\section{Materials and Methods}

Adult cats $(1.5$ to $4.0 \mathrm{~kg})$ of both sexes were anesthetized with $\alpha$-chloralose $(80 \mathrm{mg} / \mathrm{kg}$, i.p.) with few or no supplemental doses for the remainder of the experiment (12 to $18 \mathrm{hr}$ ). The animal's arterial pressure was monitored from a cannula in the carotid artery. The end-tidal $\mathrm{CO}_{2}$ was monitored and maintained within normal physiological limits (3.5 to $4.5 \%$ ) by altering either the stroke volume or the respiration rate. The animal's body temperature was monitored continuously via a rectal probe and was maintained within normal limits by external heating. At the time of the electrophysiological recordings, the animal was paralyzed with gallamine triethiodide (Flaxedil) and respired through a tracheal cannula with positive pressure. While the animal was paralyzed, the depth of anesthesia was judged by monitoring blood pressure and pupillary constriction. Flaxedil was also allowed to wear off periodically to ensure anesthesia. Microelectrodes were broken micropipettes filled with 3 $\mathrm{M} \mathrm{KCl}$ measuring 5 to 10 megohms.

Acutely and chronically deafferented preparations were subjected to identical rhizotomies (except as noted under "Results"). In each case the L5, L6, S1, and S2 dorsal roots were cut, leaving the $\mathrm{L} 7$ dorsal root intact. We recorded in the L6 segment in all animals and also in the L7 segment in some. Although the L7 root was intact, the L7 dorsal horn was partially deafferented, since individual dorsal roots project to neighboring segments (Brown and Culberson, 1981). However, the L7 segment undoubtedly was deafferented to a much smaller extent than was the L6 segment in L7 spared preparations (Sprague and $\mathrm{Ha}, 1964$ ). In the acute preparation, recording began immediately after the dorsal rhizotomy and lasted several hours. In three additional animals we recorded from SCT cells in the L6 segment prior to deafferentation, and then in the same segment immediately after rhizotomy, sparing L7. In the chronic preparation, recordings were made 56 to 156 days after the dorsal rhizotomy. An additional series of preparations was examined 3 to 45 days after rhizotomy to determine the time course of the changes occurring between the "acute" and "chronic" states. In each animal, the recorded segment was searched at depths up to $2 \mathrm{~mm}$ at several rostrocaudal positions from the medial border of the dorsal root entry zone, medially, to approximately the medial edge of the dorsal horn.

SCT cells were identified using the response to antidromic stimulation, according to the criteria of Brown and Franz (1969). Briefly, stimulating electrodes were placed on the dorsolateral fasciculus at $\mathrm{C} 1$ and $\mathrm{C} 3$, and the dorsal columns were cut at $\mathrm{C} 4$ to eliminate secondorder dorsal column cells (Rustioni and Kaufman, 1977). Only units responding to high frequency $(>250 \mathrm{~Hz})$, low intensity ( 2 to $10 \mathrm{~V}, 0.2 \mathrm{msec}$ ) stimulation at $\mathrm{C} 3$, which failed to respond to similar intensities of stimulation at $\mathrm{C} 1$, were accepted for analysis. After identification, the modality of the sensory input was determined using the same battery of tests for each cell. Before the sensory properties were tested, the hair on the area of the skin where the receptive fields were located was clipped to a length of approximately $1 \mathrm{~mm}$. We used methods very similar to those reported by numerous other groups
(Brown and Franz, 1969; Willis et al., 1974; Price et al., 1979; Pubols and Goldberger, 1980) to characterize, the cells. The leg was stimulated by brushing and pinching the skin in order to determine the location of the receptive field. Then, each cell was characterized as to the type of sensory input. The response to hair movement was tested first, using a short puff of air, either by blowing directly across the receptive field or by expressing air out of a Pasteur pipette. The hairs in the receptive field were then touched lightly with a fine camel's hair brush or fine cotton fibers pulled from a cotton-tipped applicator (Fig. 1). Non-noxious pressure was tested either by indenting the skin slightly with the blunt end of a brush (less than $4 \mathrm{~mm}^{2}$ rounded tip) or by compressing a fold of skin lightly with a pair of blunt forceps (less than $2 \mathrm{~mm}^{2}$ tipcontact area) at a force which was sensed as non-noxious by the experimenters. A positive response was an elevated discharge maintained throughout the duration of the stimulus (Fig. 1). Noxious stimulation was applied to the receptive field by pinching a fold of skin with tissue forceps or serrated hemostats. A positive response consisted of a clear increase in maintained firing rate beyond the level established by moderate pressure (Fig. 1). In a few cases where the increase was borderline, the cell was discarded. Although we cannot rule out a contribution from mechanoreceptors firing at higher rates or from recruitment of mechanoreceptors in surrounding skin, the requirement for a frankly noxious stimulus to achieve the highest discharge levels indicates a nociceptive input to these cells (see discussion in Price et al., 1979). Cooling the skin was achieved by applying ethyl chloride (cooling) directly to the receptive field. Care was taken to differ-
Cell 1

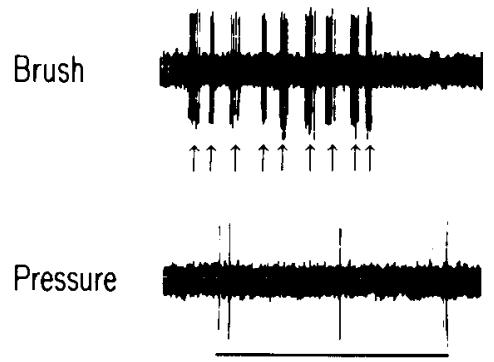

Pinch

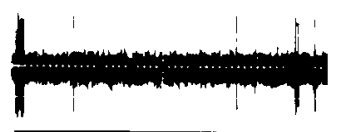

Cell 2
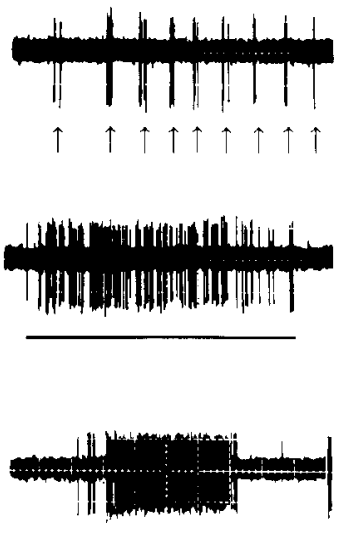

Figure 1. Response of two SCl' cells to brush, moderate pressure, and pinch. Note that Cell 1 responded to repeated hair movement elicited by brushing (arrows) with a brief discharge. When moderate pressure was applied to the receptive field by means of a blunt probe (horizontal line), the cell was virtually silent except for a transient discharge at the application and removal of the stimulus. A similar finding was made when the skin was pinched with a serrated forceps. Cell 2 was subjected to a similar regimen of stimulation. Note that it fired continuously during pressure and pinch with the rate of firing being markedly higher during pinch. The duration of each trace is $5 \mathrm{sec}$. 
entiate between the actual cooling effect of the ethyl chloride and the mechanical stimulation due to deflection of hairs (see "Discussion"). The skin was heated by means of a hand-held heating lamp until the stimulus was noxious (as judged by the subjective sensation of the experimenter, who kept his finger beside the receptive field being tested). The heating lamp was then quickly removed to eliminate any skin damage or possible sensitization. The response to this stimulus is likely to have been to noxious heat (Cervero et al., 1977).

After the modality input and receptive field size and location were determined for the cell in extracellular recording, an intracellular penetration was attempted. If this was successful, the modality input and receptive field size and location were re-examined, and these results were compared with those obtained using extracellular recording.

To carry out rhizotomy in animals requiring survival ( 3 to 156 days), the animal was anesthetized with sodium pentobarbital (Nembutal, $35 \mathrm{mg} / \mathrm{kg}$, i.p.), and using sterile technique, the dorsal root ganglia (L5 to S2) on the left side of the spinal cord were exposed by a hemilaminectomy. The dorsal roots were sectioned extradurally just proximal to the ganglion using small iridectomy scissors. Extreme care was taken not to cut the dura or damage the ventral roots. In the chronically rhizotomized animals, 250,000 units of penicillin were given immediately after surgery and for 3 to 5 days postoperatively to prevent infection. All animals recovered without incident. In "acute" preparations in which recording was carried out the same day as the deafferentation, dorsal rhizotomy was performed intradurally under the microscope, taking particular care to avoid damage to blood vessels.

\section{Results}

We recorded from a total of 374 identified SCT cells in 57 cats. These units were examined mainly in extracellular recordings, with a few also being recorded intracellularly. All of the units under the various experimental conditions except after complete unilateral deafferentation of the lumbar spinal cord responded to peripheral stimulation.

\section{Sensory modality input in the intact preparation}

In order to make a more reliable evaluation of possible changes in modality of the sensory input to spinocervical cells, it was necessary to evaluate the input to these cells in the intact spinal cord under the experimental conditions used here. The modality input to this particular population of cells has already been quite extensively studied by Brown and Franz (1969).

A total of 97 cells was examined in 15 cats using extracellular recordings. Inilially, units were characterized according to whether or not they responded to nociceptive stimulation (Mendell, 1966). Forty-five percent of the units responded only to non-noxious mechanical input, such as hair movement and/or non-noxious pressure, while $55 \%$ of the units responded to noxious mechanical input such as pinch as well as non-noxious input (see Fig. $2 A$ ). A more detailed analysis of modality input is shown in Figure $2 B$. As shown in this figure, the
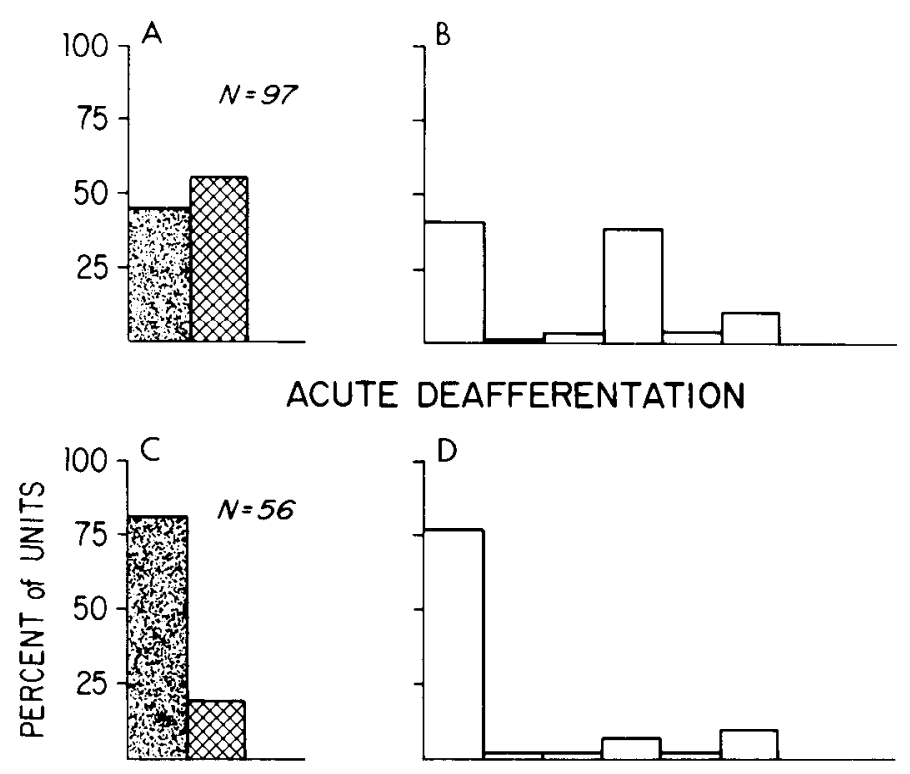

CHRONIC DEAFFERENTATION

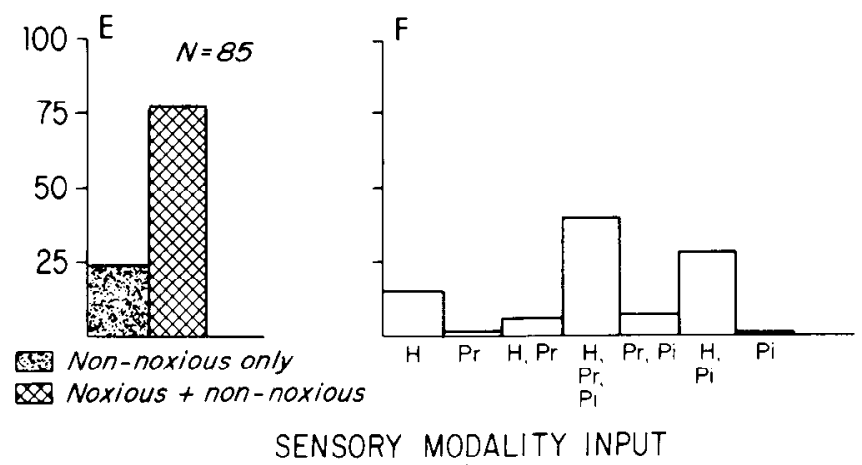

Figure 2. Sensory modality of input to SCT cells in intact and acutely and chronically deafferented preparations. $A, C$, and $E$ : Proportion of SCT units responding to non-noxious input only and to noxious + non-noxious input in intact and acutely and chronically deafferented animals, respectively. Note the change in the proportion of SCT units responding to noxious input in the chronic animals $(E)$ when compared with the acutely deafferented animals $(C), B, D$, and $F$ : Detailed analysis of sensory modality input onto SCT cells in intact and acutely and chronically deafferented animals, respectively. $N$ $=$ number of SCT units sampled under each condition. In $B, D$, and $F, H$ is hair input, $P r$ is non-noxious pressure input, and $P i$ is noxious pinch input.

majority of units responded to hair movement only (41\%) or to hair movement, non-noxious pressure, and noxious pinch (39\%). The other four categories, representing various combinations of hair movement, non-noxious pressure, and noxious pinch, occurred infrequently. Treating each modality as an independent input, we can calculate that $95 \%$ of the units responded to hair movements, $47 \%$ responded to moderate pressure, and 55\% responded to pinch in the receptive field (Table I).

The modality input and receptive field organization of 24 units were re-examined after having been successfully penetrated, with the aim of assessing the extent of subliminal inputs to SCT neurons. Based on spike activity, 
TABLE I

Percentage of SCT units responding to each mechanical stimulus in each type of preparation

Many units responded to more than one input; therefore, percentages add up to more than $100 \%$. TN $=$ total number of units sampled in each type of preparation; $N=$ number of units responding to each type of stimulus. Brackets indicate pairs of values for which the differences are statistically significant $(p<0.05)$ using the $\chi^{2}$ test Adjustment was made for the effects of multiple comparison. Note the similarity in the proportion of units responding to hair movements in the various preparations compared to the differences in proportions of neurons responding to moderate pressure or pinch.

\begin{tabular}{|c|c|c|c|}
\hline \multirow[b]{2}{*}{ Preparation } & \multicolumn{3}{|c|}{ Response of Units to } \\
\hline & $\begin{array}{c}\text { Hair } \\
\text { movement }\end{array}$ & $\begin{array}{c}\text { Moderate } \\
\text { pressure }\end{array}$ & Pinch \\
\hline Intact & $95 \%$ & $\Gamma^{47 \%}$ & $55 \%$ \\
\hline $\mathrm{TN}=97$ & $\mathrm{~N}=92$ & $N=46$ & $\mathrm{~N}=53$ \\
\hline Acute deafferentation & $96 \%$ & $13 \%$ & $20 \%$ \\
\hline $\mathrm{TN}=56$ & $\mathrm{~N}=54$ & $\mathrm{~N}=7$ & $N=11$ \\
\hline $\begin{array}{l}\text { Chronic deafferentation } \\
\mathrm{TN}=85\end{array}$ & $\begin{array}{l}89 \% \\
\mathrm{~N}=76\end{array}$ & $\begin{array}{l}55 \% \\
\mathrm{~N}=47\end{array}$ & $\begin{array}{l}\text { L } 76 \% \\
\mathrm{~N}=65\end{array}$ \\
\hline
\end{tabular}

the modality of the input was identical to that seen extracellularly. Studies of intracellular synaptic noise revealed much the same modality organization, although in units discharging only in response to hair input, we could observe a low maintained level of synaptic activity when the receptive field was pinched. We were unable to decide whether or not this was due to inadvertent weak activation of hair follicle receptors. Noxious heat stimuli could be applied without this ambiguity and in these cases, no evidence for subliminal inputs was obtained. One of these units showed a slightly larger receptive field in intracellular recording. The receptive field size of the other units was virtually unchanged. Similar findings were made in all deafferented preparations discussed below.

\section{Sensory modality input in the chronically deafferented preparation}

Once the modality of the input to a population of spinocervical units in the normal spinal cord had been established, we analyzed the sensory modality input to cells in the chronically deafferented cord. Eighty-five cells in the L6 segment were examined in 16 cats that had survived 56 to 156 days after dorsal roots L5, L6, S1, and $S 2$ had been sectioned. Figure $2 E$ demonstrates that the proportion of units responding only to non-noxious input decreased from $45 \%$ to $24 \%$, whereas the proportion of units responding to noxious input increased from $55 \%$ to $76 \%$. The more detailed analysis of the types of modality input is represented in Figure $2 F$. The majority of spinocervical cells in the chronic cord now responded to a combination of hair movement, non-noxious pressure, and noxious pinch $(40 \%)$ or hair movement and noxious pinch (28\%). From Table I, it can be seen that the percentage of units responding to hair movement and moderate pressure was similar to that found in the intact preparation. However, the percentage of units sensitive to nociceptive inputs increased from $55 \%$ to $76 \%$, which was significant $(p<0.05$; see Table I).

\section{Sensory modality input in the acutely deafferented preparation}

Comparison of modality input in chronically deafferented and intact preparations requires that the L7 spared root provides a spectrum of modality input to SCT neurons in the L6 segment that is similar to the one provided by the normal complement of afferent fibers (i.e., all roots intact). To evaluate this, we have examined the responses of SCT neurons in L7 spared root preparations subjected to rhizotomy on the day of the experiment.

Fifty-six units were examined in eight cats within the first few hours after the L5, L6, S1, and S2 dorsal roots had been sectioned intradurally. Under these conditions, $81 \%$ of the units examined responded only to non-noxious input (Fig. $2 C$ ), compared to $45 \%$ in the intact preparation (Fig. $2 A$ ). In three additional cats in which recordings were made from the same segments before and immediately after partial rhizotomy, the proportions changed from $42 \%$ non-nociceptive input only ( $58 \%$ nonnociceptive + nociceptive) in the intact preparation $(N$ $=26$ ) to $78 \%$ non-nociceptive input only ( $22 \%$ non-nociceptive + nociceptive) after deafferentation $(N=27)$. Closer examination (Fig. 2D) showed that the major change from the intact preparation was a loss of units responding to hair, pressure, and noxious pinch and a corresponding gain of units responding to hair input alone. Notably, there was little increase in the proportion of units responsive to pressure only or to hair and pressure. From Table I, it can be seen that $96 \%$ of the units in acutely deafferented L6 were sensitive to hair movements, which was similar to the intact preparation. In contrast, only $13 \%$ and $20 \%$ were responsive to pressure and pinch, respectively, a significant decrease from the values found in the intact preparation (Table I). The increases in the proportion of units responding to pinch after chronic deafferentation were much larger when compared to the acutely deafferented preparation than to the intact one (Table I). Furthermore, the comparison of "chronic" to "acute" revealed an increase in the percentage of SCT neurons responsive to moderate pressure, although this is not evident by consideration of "chronic" and "intact" preparations (Table I). These findings demonstrate that the acutely deafferented cord is a better control for the evaluation of the effects of chronic rhizotomy than is the intact cord.

\section{Responses to skin temperature changes}

Responses to thermal stimulation (noxious heat, cooling) were examined in intact and in acutely and chronically deafferented preparations. In intact preparations, thermal input was tested in 81 of the 97 units analyzed, and $64 \%$ of these were found to respond to either cooling or heating of the skin or, in many cases, both. No significant differences were found in the different preparations in the proportions of units responding to cooling and to noxious heating of the skin; therefore, these responses are combined (Fig. 3). In the chronically deafferented preparations, 62 (of 85) units were tested for sensitivity to skin temperature changes and $68 \%$ of these ( 42 of 62 ) responded to either cooling or heating or both. Exami- 


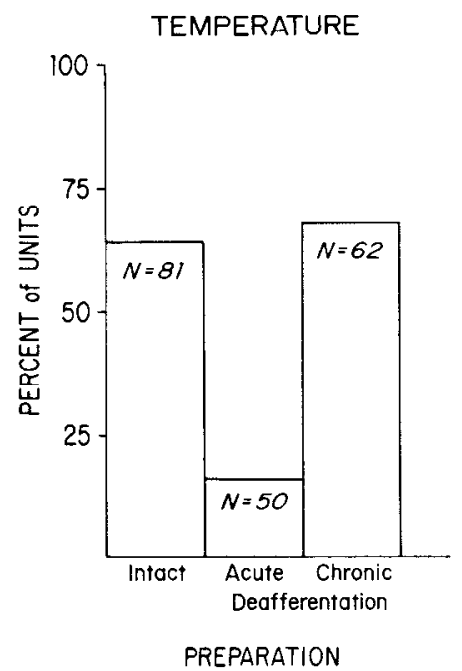

Figure 3. Changes in thermal sensitivity of SCT cells after acute and chronic deafferentation. After acute deafferentation there was a decrease in the proportion of units responding to temperature changes. Chronically deafferented animals showed an increase in the proportion of SCT units responding to thermal input. $N=$ number of SCT units for which responses to temperature changes were evaluated.

nation of the intact and chronic preparations showed that the percentages of units responding to temperature changes were about the same (Fig. 3). As mentioned above, we felt that the more appropriate comparison was between responses in chronically and acutely deafferented preparations. In the acutely deafferented preparation, 50 of 56 units were tested for thermal input. Only 8 $(16 \%)$ of these units responded to temperature changes (Fig. 3). Thus, it can be seen that after chronic deafferentation, a significant increase occurred in the percentage of units responding to changes in skin temperature. This increase in response to temperature changes parallels the increase in responsiveness to nociceptive and moderate pressure input.

\section{Sensory modality input to neurons in the L7 (spared root) segment}

In 9 of the 16 chronically deafferented animals, recordings were also made from the L7 (spared root) segment. Twenty-six units were examined. Figure $4 A$ shows that the proportions of units responding to non-noxious and noxious input, and to non-noxious input only, were similar to those found in the intact preparation (see Fig. $2 A$ ). This suggests that the spinocervical cells in the segment whose primary afferent input has been left relatively intact respond normally.

Similar recordings were made from the intact L7 segment in the acutely deafferented preparation. Sixteen units were examined in four of the eight acutely deafferented animals. Figure $4 B$ shows again that the proportion of cells responding to non-noxious and noxious inputs, and non-noxious input only were very similar to the responses found in the intact preparation (see Fig. 2A). These results indicate that the alterations were restricted to the deafferented segment and were not a result of more generalized changes in the animal. They also con-

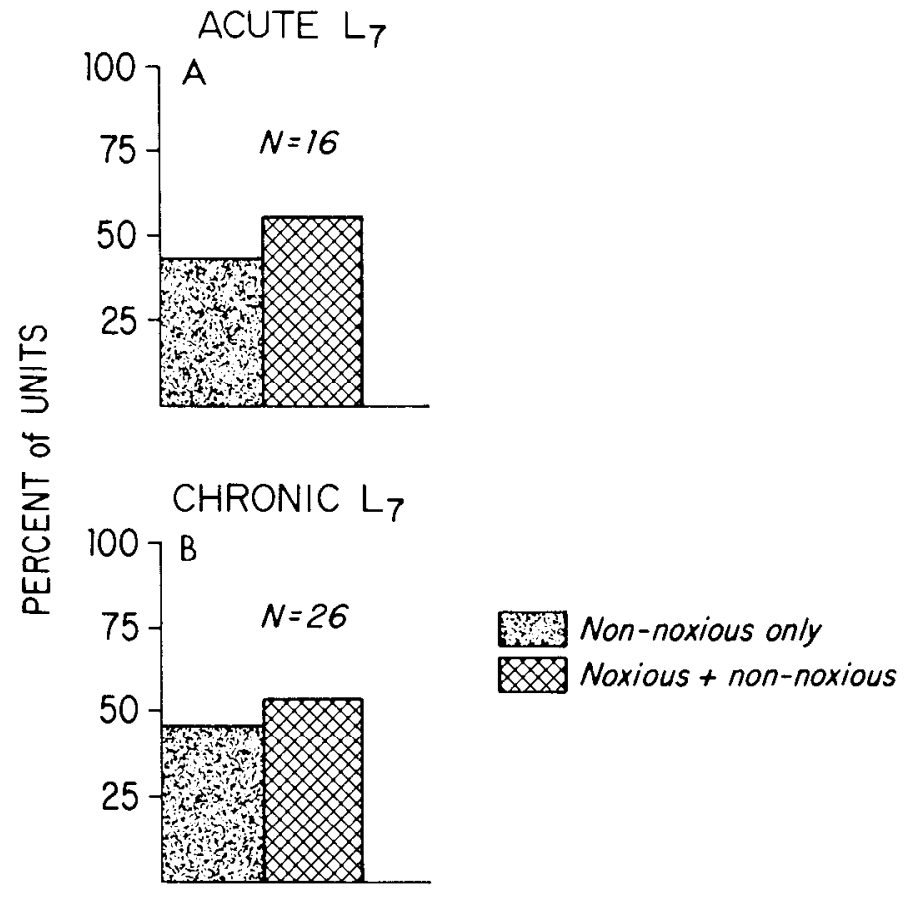

\section{SENSORY MODALITY INPUT}

Figure 4. Sensory modality input onto SCT cells in the L7 (spared root) segment after acute and chronic rhizotomy. The proportion of cells responding to non-noxious and noxious input and non-noxious input only are similar to the responses in the intact preparation. $N=$ number of SCT units sampled.

firm that the central changes we have observed are not due to changes in the properties of the cutaneous terminals of the surviving afferent fibers.

\section{Complete deafferentation of the lumbar enlargement}

Nineteen SCT units in the L6 segment of three cats were examined after a complete dorsal rhizotomy of L5 to S2. Twelve of these units responded to stimulation of skin regions outside the usual dermatome. The other seven units had no receptive field. The receptive fields, when present in these preparations, were usually larger than the fields for units in the intact cord and were located in the proximal portion of the hindlimb. In some cases the receptive fields extended over the abdominal region to the rib cage. These unusual receptive fields are similar to those described by Basbaum and Wall (1976) and Mendell et al. (1978). They were not seen in the preparations described above in which $L 7$ was spared. The surviving L7 dorsal root evidently prevents the development of input from the more rostral segments onto neurons of L7, presumably because these neurons are not sufficiently deafferented.

Eleven of the units in the completely deafferented animals responded to hair movement only, while one unit responded to hair movement, non-noxious pressure, and noxious pinch. The response of these units to hair movement was much different from that of units found in the intact or partially deafferented cord. They responded to very gentle brushing only and habituated very quickly to rapid brushing. These units did not habituate when the 
receptive field was brushed very slowly ( 1 stroke/sec or slower). No such units were found in the spared root preparations.

\section{Time course of modality changes in acute and chronic preparations}

We were interested in defining the time at which the spinocervical units increased their probability of responding to nociceptive inputs. Ninety-five units were examined in 15 cats at approximately 1 -week intervals from 3 days to 45 days after the partial deafferentation. At all intervals up to 39 days, most units responded to hair input only, and few could be activated by nociceptive inputs (Fig. 5). Only at 45 days did a larger proportion of units exhibit sensitivity to nociceptive input in the manner described above as characteristic of chronically deafferented preparations. It appears, therefore, that the change of modality input (i.e., acquisition of nociceptive inputs) in spinocervical cells in the partially deafferented spinal cord occurs at about 45 days. This timing differs from that reported by Basbaum and Wall (1976), who stated that the numbers of newly connected cells seemed to have been established by one month after the partial deafferentation. This may reflect differences in the reorganization of somatotopy and modality convergence following partial deafferentation.

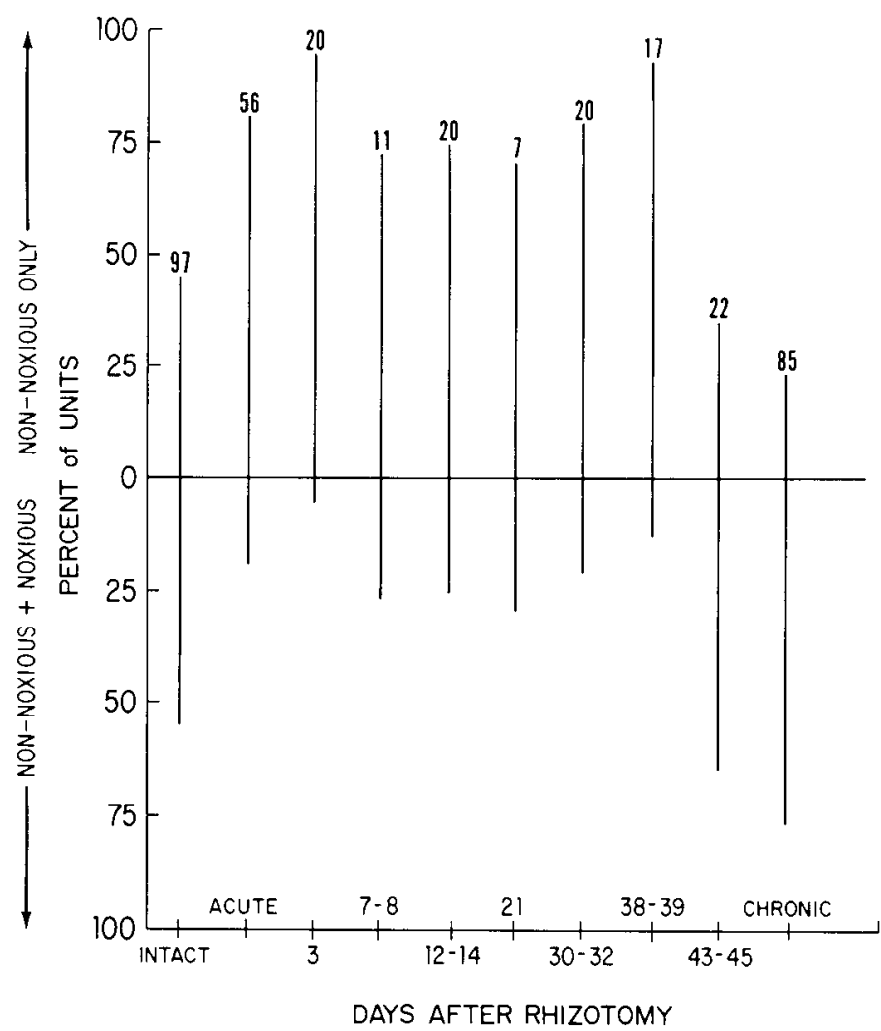

Figure 5. Time course of alteration of modalities of SCT cells after partial rhizotomy. At all intervals up to day 39 , most cells responded to non-noxious input only. Beyond that time, most SCT cells responded to non-noxious input and increased their firing rate during maintained noxious stimulation. The number of SCT units sampled during each time interval is shown above each line.

\section{Discussion}

From the display in Figure 5, it might be concluded that the only change following deafferentation occurred in the period beginning immediately after rhizotomy and lasted about 6 weeks, and that the alterations which we have termed "chronic" might in fact have been nothing more than a return to normal. This seems unlikely for the following reasons. First, on the assumption that the acute change was the result of the injury (e.g., some form of "shock"), recovery should have occurred relatively quickly, within days, as has been shown after spinal transection in the cat (Murray and Goldberger, 1974) or after dorsal rhizotomy (Pubols and Goldberger, 1980), rather than in weeks, as we have shown. When this line of reasoning is combined with the finding of the abruptness and stability of the change after acute rhizotomy, the conclusion which emerges is that the acute changes would probably have been permanent but for the emergence of the chronic changes. Furthermore, when the chronic changes finally did emerge after 6 weeks, there was a significant increase over the intact preparation in the proportion of cells responsive to noxious mechanical input ( $76 \%$ versus $55 \%$, Table I). In other words, the chronically deafferented preparation is different from the intact one, particularly with regard to the proportion of SCT cells responsive to noxious mechanical inputs. All of these considerations lead to the assertion that the changes after chronic rhizotomy must be evaluated in terms of the stable changes occurring after acute rhizotomy.

In the current study, all units were rigorously identified as spinocervical cells by antidromic activation. This sampling method eliminates the possibility of bias from abnormally active or "silent" cells which is introduced when units are located by their spontaneous activity. Furthermore, the use of an identified population of cells is important in assessing their inputs (see below) since these differ depending on the destination of their axon, even if cell bodies are in the same general position of the dorsal horn (Brown, 1981).

It seems unlikely that sampling could have inadvertently selected cells of different types in the acutely and chronically deafferented segments. In each animal, the L6 segment was searched in a systematic way (see "Materials and Methods") with care being taken not to favor one region over another. We found that in the intact segment there was no correlation between the position of a cell in the dorsal horn and its sensory modality input. This also held true for both the chronically and acutely deafferented segments. We feel, therefore, that the changes in modality input after the acute and chronic rhizotomies were not due to sampling bias.

Our major finding is that partial deafferentation of cat spinal cord causes a change in the modality of the sensory input to SCT neurons in the denervated segment. In intact preparations, $45 \%$ of the SCT neurons we recorded from responded to non-noxious mechanical input only. In acutely deafferented spinal segments, this proportion increased to $81 \%$, occurring principally as an increase in the proportion of neurons sensitive to hair input only. In chronically deafferented preparations, the proportion of SCT cells affected by non-noxious inputs only fell to $24 \%$, 
primarily because the proportion of cells responding to pinch (i.e., $\mathrm{H}+\mathrm{Pi}$; $\mathrm{H}+\mathrm{Pr}+\mathrm{Pi}$; $\mathrm{Pr}+\mathrm{Pi}$ ) increased (Fig. 2). The increase in nociceptive SCT cells was paralleled by an increase in their response to cooling and noxious heat. This increase in the degree of responsiveness to noxious mechanical al.d thermal stimulation occurred rather abruptly between 38 and 45 days after surgery. Results consistent with these have been reported by Pubols and Brenowitz (1982).

A more detailed treatment of input modality (Table I) reveals no change in responsiveness of SCT cells to hair displacement in the three groups of animals, although we cannot be certain that more subtle changes in the input from the different kinds of hair follicle receptors (Brown and Iggo, 1967; Burgess et al., 1968) did not occur. In contrast, input from mechanical nociceptors (pinch) exhibited a decrease in the acutely deafferented preparation followed by an increase in the chronically deafferented preparation. The response to moderate pressure, cooling, and noxious heat also exhibited changes in these three states which paralleled those displayed by responses to pinch. Thus, the probability that SCT cells respond to some modalities (pinch, moderate pressure, noxious heat, cooling) changes during the different stages that follow dorsal rhizotomy.

It is impossible at present to decide the basis for the differences in response to deafferentation on the part of inputs from hair receptors, on the one hand, and inputs from pressure, mechanical nociceptive, noxious heat, and cooling receptors, on the other. In consideration of this question we would like to raise the possibility that these two classes of inputs to SCT neurons differ according to the diameter of the afferent fibers that innervate the receptors. Previous work has shown the SCI neurons receive input from hair receptors and various nociceptors and thermoreceptors, but not from slowly adapting low threshold mechanoreceptors whose axons conduct in the A $\beta$ velocity range (e.g., type I and type II) (Brown and Franz, 1969). Thus the responses to moderate pressure, at least in the intact and acutely deafferented preparations and possibly in the chronically deafferented preparation (see below), result from the action of relatively luw threshold mechanoreceptors projecting mainly via A $\delta$ fibers (Burgess and Perl, 1967; Burgess et al., 1968) or unmyelinated fibers (Douglas and Ritchie, 1957; Iggo, 1960; Bessou et al., 1971). This would also be true for mechanical nociceptors (Burgess and Perl, 1967; Perl, 1968; Beitel and Dubner, 1976; Kumazawa and Perl, 1977), noxious thermoreceptors (Iggo, 1959; Bessou and Perl, 1969; Beck et al., 1974; Croze et al., 1976; Georgopoulos, 1976), and, to a great extent, cold receptors (Iggo, 1959; Hensel et al., 1960; Iggo, 1969; Dubner et al., 1975). On the other hand, hair receptors project to a large degree via large myelinated $\mathrm{A} \beta$ fibers (Brown and Iggo, 1967; Burgess et al., 1968; Brown, 1981). The important conclusion is that the responses of SCT cells to pressure, pinch, noxious heat, and cold, which changed after deafferentation, are mediated primarily by $\mathrm{A} \delta$ and $\mathrm{C}$ fiber inputs, whereas responses to hair movements which were stable are mediated mainly by inputs arriving over large fiber $(\mathrm{A} \beta)$ inputs.

The changes following acute partial deafferentation may result from selective interruption of afferent fibers conducting impulses from receptors that mediate sensitivity to maintained pressure and nociception. This may be a consequence of these modalities being transmitted to SCT cells primarily by small diameter $(\mathrm{A} \delta, \mathrm{C})$ afferents in contrast to inputs from hair receptors, which are conducted via large myelinated (A $\beta$ ) afferents (see above). Similar considerations apply to evaluation of the inputs from cold and noxious heat thermal receptors. Parenthetically, it is worth noting that the decrease in response to skin cooling after acute deafferentation, despite maintenance of normal input from hair receptors, indicates that the cooling stimulus used did not influence the cells via hair movements (see "Materials and Methods"). The rostrocaudal extent of small diameter afferent fibers (Wall and Yaksh, 1978) may be more limited than that of large diameter fibers (Brown, 1981). If SCT neurons in L6 received the majority of their nociceptive, pressure, and thermal input from afferent fibers entering through the L6 dorsal root and if hair inputs were derived from more wide-ranging afferents (i.e., from L7 as well as L6), cutting L6 and leaving L7 intact would deprive the $\mathrm{L} 6$ segment of a disproportionate amount of maintained pressure, nociceptive, and thermal input. In accordance with this hypothesis, we found that the proportion of units responsive to nociceptive input in the L7 segment that remained innervated was similar to that in the intact preparation (Fig. 1A) (but sec Pubols and Brenowitz, 1982). However, there is some evidence that small fibers project through Lissauer's tract to neighboring segments (Chung and Coggeshall, 1979) and, therefore, these differences may have a functional rather than an anatomical basis. Thus, it remains possible that these acute changes result from as yet undefined alterations in transmission through interneurons (e.g., in the marginal zone or substantia gelatinosa; Price et al., 1979).

One possible mechanism for the changes we have seen in the chronic spinal cord is sprouting of primary afferents (Liu and Chambers, 1958; Goldberger and Murray, 1974). The pattern of the changes we have observed is suggestive of selective sprouting of terminals of small myelinated and unmyelinated axons normally associated with the transmission of pressure, nociceptive, and thermal information from the periphery (Burgess and Perl, 1967; Iggo, 1960; Bessou and Perl, 1969). There is no evidence at present that small fibers sprout preferentially in the spinal cord, and we cannot say that the acquisition of moderate pressure sensitivity did not result from sprouting of $\mathbf{A} \beta$ fibers mediating input from type I and type II slowly adapting mechanoreceptors. However, it may be relevant that when the skin of adult rats is denervated, sprouting of intact nerve fibers is restricted in large part to small fibers that innervate high threshold mechanoreceptors (Jackson and Diamond, 1979).

The spinal pathways mediating these changes are presently unknown. Since the marginal zone and substantia gelatinosa have been shown to be an important relay for input from small diameter afferents into the spinal cord (Christiansen and Perl, 1970; Kumazawa and Perl, 1978; Light et al., 1979; Price et al., 1979), it is conceivable that increased relay of inputs through marginal zone or sub- 
stantia gelatinosa cells to SCT cells may account for enhanced responses which we have observed. Alternatively, these new pathways might bypass the superficial layers of the dorsal horn and involve the projection of small diameter afferent fibers to SCT neurons.

Enhancement in the efficacy of previously subliminal input onto chronically deafferented SCT cells (see Mendell et al., 1978) seems unlikely because intracellular recordings in intact preparations did not reveal a subthreshold thermal nociceptive input in units classified as responding to hair only in extracellular recording. However, we cannot rule out the possibility that this might have occurred on spinal neurons which themselves project to SCT neurons (e.g., in the marginal zone or substantia gelatinosa).

Since transmission through the SCT is inhibited by impulses arriving over afferent (Brown et al., 1975) and several descending pathways including the dorsolateral funiculi, the most medial and ventral parts of the ventral funiculus and dorsal columns (Brown et al., 1973), it is conceivable that dorsal rhizotomy as performed in our experiments might enhance the response of SCT cells by diminishing the magnitude of this inhibitory control. Against this interpretation is our finding that the changes began only 6 weeks after rhizotomy; this does not support the disinhibition hypothesis which would predict immediate increases in transmission from the periphery. Furthermore, an increase in the moderate pressure, nociceptive, and thermal input in the intact $\mathbf{L} 7$ segment might have been anticipated inasmuch as there is considerable overlap in the somatotopy of the L6 and L7 segment, but this was not observed.

We conclude that partial deafferentation of the cat dorsal horn causes substantial reorganization in the modality of the inputs to the cells of the SCT. This reorganization is particularly evident when the chronically deafferented preparation is compared to the acutely deafferented preparation rather than to the intact one. However, we feel this is the appropriate comparison since the functional projection of fibers mediating the various modalities is systematically different based on our comparison of intact and acutely partially deafferented preparations. The sensory consequences of having larger numbers of SCT neurons sensitive to moderate pressure, nociceptive, and thermal inputs is not known. It would depend in part on changes which occur on other neurons that influence forebrain function. It seems possible, then, that in the weeks following partial deafferentation, forebrain structures normally driven by the SCT system might become increasingly dominated by input arriving over small diameter peripheral fibers. This might have the effect of lowering the threshold for pain from the affected dermatome or perhaps changing its perceived quality. Verification of these speculations will require behavioral studies.

\section{References}

Basbaum, A. I., and P. D. Wall (1976) Chronic changes in the response of cells in the adult cat dorsal horn following partial deafferentation: The appearance of responding cells in a previously nonresponsive region. Brain Res. 116: 181-204.

Beck, P. W., H. O. Handwerker, and M. Zimmermann (1974) Nervous outflow from the cat's foot during noxious radiant heat stimulation. Brain Res. 67: 373-386.

Beitel, R. E., and R. Dubner (1976) Response of unmyelinated (C) polymodal nociceptors to thermal stimuli applied to monkey's face. J. Neurophysiol. 39: 1160-1175.

Bessou, P., and E. R. Perl (1969) Response of cutaneous sensory units with unmyelinated fibers to noxious stimuli. J. Neurophysiol. 32: 1025-1043.

Bessou, P., P. R. Burgess, E. R. Perl, and C. B. Taylor (1971) Dynamic properties of mechanoreceptors with unmyelinated (C) fibers. J. Neurophysiol. 34: 116-131.

Brown, A. G. (1981) Organization in the Spinal Cord: The Anatomy and Physiology of Identified Neurones, SpringerVerlag, New York.

Brown, A. G., and D. N. Franz (1969) Responses of spinocervical tract neurones to natural stimulation of identified cutaneous receptors. Exp. Brain Res. 7: 231-249.

Brown A. G., and A. Iggo (1967) A quantitative study of cutaneous receptors and afferent fibres in the cat and rabbit. J. Physiol. (Lond.) 193: 707-733.

Brown, A. G., E. J. Kirk, and H. F. Martin, III (1973) Descending and segmental inhibition of transmission through the spinocervical tract. J. Physiol. (Lond.) 230: 689-705.

Brown, A. G., W. C. Hamann, and H. F. Martin, III (1975) Effects of activity in non-myelinated afferent fibres on the spinocervical tract. Brain Res. 98: 243-259.

Brown, P. B., and J. L. Culberson (1981) Somatotopic organization of hindlimb cutaneous dorsal root projections to cat dorsal horn. J. Neurophysiol. 45: 137-143.

Burgess, P. R., and E. R. Perl (1967) Myelinated afferent fibres responding specifically to noxious stimulation of the skin. J. Physiol. (Lond.) 190: 541-562.

Burgess, P. R., D. Petit, and R. M. Warren (1968) Receptor types in cat hairy skin supplied by myelinated fibers. J. Neurophysiol. 31: 833-848.

Cervero, F., A. Iggo, and V. Molony (1977) Responses of spinocervical tract neurones to noxious stimulation of the skin. J. Physiol. (Lond.) 267: 537-558.

Christiansen, B. N., and E. R. Perl (1970) Spinal neurons specifically excited by noxious or thermal stimuli: Marginal zone of the dorsal horn. J. Neurophysiol. 33: 293-307.

Chung, K., and R. E. Coggeshall (1979) Primary afferent axons in the tract of Lissauer in the cat. J. Comp. Neurol. 186: 451464.

Croze, S., R. Duclaux, and D. R. Kenshalo (1976) The thermal sensitivity of the polymodal nociceptors in the monkey. J. Physiol. (Lond.) 263: 539-562.

Douglas, W. W., and J. M. Ritchie (1957) Non-medullated fibres in the saphenous nerve which signal touch. J. Physiol. (Lond.) 139: $385-399$

Dubner, R., R. Sumino, and W. I. Wood (1975) A peripheral "cold" fiber population responsive to innocuous and noxious thermal stimuli applied to monkey's face. J. Neurophysiol. 38: 1373-1389.

Georgopoulos, A. P. (1976) Functional properties of primary afferent units probably related to pain mechanisms in primate glabrous skin. J. Neurophysiol. 39: 71-83.

Goldberger, M. E., and M. Murray (1974) Restitution of function and collateral sprouting in the cat spinal cord: The deafferented animal. J. Comp. Neurol. 158: 37-54.

Hensel, H. H., A. Iggo, and I. Witt (1960) A quantitative study of sensitive cutaneous thermoreceptors with $\mathrm{C}$ afferent fibres. J. Physiol. (Lond.) 153: 113-126.

Iggo, A. (1959) Cutaneous heat and cold receptors with slowly conducting (C) afferent fibres. Q. J. Exp. Physiol. 44: 362370 .

Iggo, A. (1960) Cutaneous mechanoreceptors with afferent C fibres. J. Physiol. (Iond.) 152: 337-353.

Iggo, A. (1969) Cutaneous thermoreceptors in primates and sub-primates. J. Physiol. (Lond.) 200: 403-430. 
Jackson, P. C., and J. Diamond (1979) Is sensory nerve activity necessary for collateral sprouting in the skin of adult rats? Soc. Neurosci. Abstr. 5: 628.

Kumazawa, T., and E. R. Perl (1977) Primate cutaneous sensory units with unmyelinated $(\mathrm{C})$ afferent fibers. J. Neurophysiol. 40: 1325-1338.

Kumazawa, T., and E. R. Perl (1978) Excitation of marginal and substantia gelatinosa neurons in the primate cord: Indications of their place in dorsal horn functional organization. J. Comp. Neurol. 177: 417-434.

Light, A. R., D. L. Trevino, and E. R. Perl (1979) Morphological features of functionally defined neurons in the marginal zone and substantia gelatinosa of the spinal dorsal horn. J. Comp. Neurol. 186: 151-172.

Liu, C. N., and W. W. Chambers (1958) Intraspinal sprouting of dorsal horn axons. A.M.A. Arch. Neurol. Psychiatry 79: 4661.

Mendell, L. M. (1966) Physiological properties of unmyelinated fiber projection to the spinal cord. Exp. Neurol. 16: 316-331.

Mendell, L. M., E. M. Sassoon, and P. D. Wall (1978) Properties of synaptic linkage from long ranging afferents onto dorsal horn neurones in normal and deafferented cats. J. Physiol. (Lond.) 285: 299-310.

Murray, M., and M. E. Goldberger (1974) Restitution of function and collateral sprouting in the cat spinal cord: The partially hemisected animal. J. Comp. Neurol. 158: 19-36.

Perl, E. R. (1968) Myelinated afferent fibres innervating the primate skin and their responses to noxious stimuli. J. Physiol. (Iond.) 1.97: 593-615.

Price, D. D., H. Hayashi, R. Dubner, and M. A. Ruda (1979)
Functional relationships between neurons of marginal and substantia gelatinosa layers of primate dorsal horn. J. Neurophysiol. 42: 1590-1608.

Pubols, L. M., and G. L. Brenowitz (1982) Maintenance of dorsal horn somatotopic organization and increased highthreshold response after single-root or spared-root deafferentation in cats. J. Neurophysiol. 47: 103-112.

Pubols, L. M., and M. E. Goldberger (1980) Recovery of function in dorsal horn following partial deafferentation. J. Neurophysiol. 43: 102-117.

Rustioni, A., and A. B. Kaufman (1977) Identification of cells of origin of non-primary afferents to the dorsal column nuclei of the cat. Exp. Brain Res. 27: 1-14.

Sedivec, M. J., J. Ovelmen-Levitt, and L. M. Mendell (1981) Changes in modality convergence in cells of partially deafferented dorsal horn. In Spinal Cord Sensation, A. G. Brown and M. Rethelyi, eds., pp. 329-330, Scottish Academic Press, Edinburgh.

Sprague, J. M., and H. Ha (1964) The terminal fields of dorsal fibers in the lumbosacral spinal cord of the cat and the dendritic organization of the motor nuclei. Prog. Brain Res. 11: $120-152$.

Wall, P. D., and T. L. Yaksh (1978) Effect of Lissauer tract stimulation on activity in dorsal roots and in ventral roots. Exp. Neurol. 60: 570-583.

Willis, W. D., D. L. Trevino, J. D. Coulter, and R. A. Maunz (1974) Responses of primate spinothalamic tract neurons to natural stimulation of hindlimb. J. Neurophysiol. 37: 358372. 